\title{
Opinions of healthcare providers on the design of a smartphone application for asthma treatment in the Kingdom of Saudi Arabia
}

\author{
Hajed M. Al-Otaibi, ${ }^{1}$ Khalid A. Ansari, ${ }^{2}$ Osama Hamad, ${ }^{3}$ Turki M. Alanzi ${ }^{3}$ \\ ${ }^{1}$ Department of Respiratory Therapy, Faculty of Medical Rehabilitation Sciences, King Abdulaziz, University, Jeddah, Saudi \\ Arabia \\ ${ }^{2}$ Department of Health Sciences, University of the West of England (UWE), Bristol, UK \\ ${ }^{3}$ Health Information Management and Technology Department, College of Public Health, Imam Abdulrahman Bin Faisal \\ University, Dammam, Saudi Arabia
}

\begin{abstract}
Introduction: In several countries of the world, smartphone applications have been designed to contribute to the treatment of asthma. However, none of these applications has been developed in Saudi Arabia. Therefore, the objective of this article is to design a smartphone application for the treatment of asthma based on the opinions of healthcare providers from the Kingdom of Saudi Arabia.

Methods: In order to know the opinion of the healthcare providers from Saudi Arabia about the design of an asthma App, we used a purposive sampling method and conducted a cross sectional survey employing a questionnaire which was distributed through the QuestionPro.com website to all healthcare providers working in this country. The questionnaire was sent to 376 healthcare providers and the response rate was $25 \%$.

Results: The data indicated that the majority of the respondents opined that the following features were important or very important in the design of a smartphone application for asthma treatment in Saudi Arabia: information about patient diagnosis (98\%), primary physician access information(83\%), patient satisfaction with the therapeutic process (91\%), push notifications about reminder for drugs (95\%), push notification for treatment of inhaler and other drugs (92\%), push notifications about reminders of clinic visits and therapy sections (81\%), push notifications to ask for help sending SMS to primary physician about patients' attacks (89\%), pathophysiology of asthma (82\%), asthma triggers $(98 \%)$, drug guidelines (94\%), drug side effects (93\%), number of asthma attacks (98\%), medication statistics $(88 \%)$, visual inputs such as peak flow $(91 \%)$, data to link patients to healthcare providers and to healthcare centers $(82 \%)$, and Global Initiative for Asthma (GINA) references (72\%).

Conclusions: According to the opinion of the majority of healthcare providers (92\%), the proposed smartphone application designed based on medical guidelines will contribute to improve the treatment of patients with asthma in the Kingdom of Saudi Arabia, and will help to reduce the number of asthma cases that need hospitalization, and the number of asthma cases in the emergency departments of the hospitals of the Kingdom.
\end{abstract}

Key words: Design; smartphone application; asthma; healthcare providers; Saudi Arabia.

Correspondence: Turki M. Alanzi, Department of Health Information Management and Technology, College of Public Health, Imam Abdulrahman Bin Faisal University, King Faisal Road, Dammam 31441, Saudi Arabia.

Tel +966.133331211 - Fax: +966.133331211. E-mail talanzi@iau.edu.sa

Contributions: All the authors made a substantive intellectual contribution. All the authors have read and approved the final version of the manuscript and agreed to be accountable for all aspects of the work.

Conflict of interest: The authors declare that they have no competing interests, and all authors confirm accuracy.

Funding: Not applicable

Availability of data and material: The data used to support the findings of this study are available from the corresponding author upon request.

Ethics approval and consent to participate: The completion of the questionnaire was considered to imply informed consent to participate in the study, and the ethical approval was obtained from the Institutional Review Board of the Imam Abdulrahman Bin Faisal University.

Consent for publication: Not applicable. 


\section{Introduction}

According to the Global Initiative for Asthma 2019 Report (GINA 2019), asthma is defined as a "heterogeneous disease characterized by chronic airway inflammation" [1]. In addition, this illness is typified by "the history of respiratory symptoms such as wheeze, shortness of breath, chest tightness and cough that vary over time and in intensity together with variable expiratory air flow limitation" [1]. Currently, there are around 339 million people in the world affected by this heterogeneous respiratory disease [2].

To manage this illness, in addition to conventional treatments, new alternatives based on the so-called m-health technologies which use communications, information, and mobile technologies advancements, have been implemented in order to provide medical care remotely [3]. Some of these alternatives employ software applications developed specifically for use on small, wireless computing devices, such as smartphones and tablets, rather than desktop or laptop computers [4].

In relation to asthma, a recent study identified 523 applications available in the Apple app store and Google Play store [5]. Most of these applications have been developed in the United States (37\%), Switzerland (11\%), Spain (5\%), India (5\%), Australia (5\%), the United Kingdom (5\%), Germany (3\%), Portugal (3\%), the Czech Republic (3\%), Lithuania (3\%), Latvia (3\%) and other countries $(17 \%)$ [5]. The largest proportion of these applications have been developed by private companies and independent developers; and the rest by organizations related to healthcare, university hospitals, non-profit organizations and government entities [5]. According to some authors, several of these applications are suitable for asthma self-management, but most of them are of low quality and are not based on medical and clinical guidelines [5]. Similarly, other studies have suggested that these technologies are effective in helping the management and treatment of asthma [6-11]. For example, one study reported that a smartphone app used continuously for 4 months was effective in improving asthma control in a group of 60 adult patients [7]. Another study showed that the use of a smartphone application contributed to improve the control of asthma in a group of adolescents with persistent asthma [9].

As for Saudi Arabia, statistics indicated that there is a high prevalence of asthma in adults, which is higher than most of the countries reported in the European Community Respiratory Health Survey (ECRHS), and in general its incidence in the Kingdom varies between $15-25 \%$ per region $[12,13]$. Perceptibly, this high percentage of patients has to be followed through the use of smartphone applications and social media tools that can contribute to improve asthma management and reduce the number of affected cases and side effects through awareness and direct communication. Parallel to this situation, Saudi Arabia with an estimated population of more than 33 million, has the highest penetration rate of smartphones in the region, and by 2018 the number of smartphone users was about 22 million [14-16]. Despite the high prevalence of asthma in the Kingdom of Saudi Arabia and the impressive use of smartphones in the region, the review of the literature showed that no mobile application for the treatment of asthma had been developed in the Kingdom.

For these reasons, the purpose of this paper is to design a smartphone application for the treatment of asthma based on the medical opinions of a group of healthcare providers from the Kingdom of Saudi Arabia.

\section{Methods}

\section{Study settings and participants}

To carry out this study, we used a purposive sampling method and selected a sample of young healthcare providers easily accessible and specialized in respiratory diseases in the Kingdom of Saudi Arabia. According to some studies, young doctors are the ones who use smartphone applications most frequently in their professional practices $[17,18]$. In our case, the sample of selected young doctors also had good knowledge about the characteristics of asthma disease due to their specialization. In other words, these surveyed doctors are the ones who can give us the most appropriate and convincing opinion about the design of a smartphone application for asthma treatment in Saudi Arabia. For this reason, other respondents with a wider age range and professional experience were not considered. Then, we sent to all healthcare providers chosen in the Kingdom of Saudi Arabia a cross sectional survey questionnaire which was prepared by the research group. The questionnaire was distributed through the QuestionPro.com website. Also, hardcopies were circulated to 3 different sectors in the eastern region of this country (King Fahd University Hospital, King Fahad Specialist Hospital at Dammam, and Prince Sultan Military College of Health Sciences). Alike, the direct link for the questionnaire was distributed throughout WhatsApp and other smartphone applications. The survey was distributed among 376 selected healthcare providers, but only 94 of them $(25 \%)$ responded to the survey. The data was collected between January and February 2018.

The completion of the questionnaire was considered to imply informed consent to participate in the study, and the ethical approval was obtained from the Institutional Review Board of the Imam Abdulrahman Bin Faisal University.

\section{Description of the questionnaire}

The survey questionnaire had 2 parts and contained some "yes" or "no" questions, and several questions directed to assess the level of importance expressed in terms of the following scale: "not important", "somewhat important", "important" and "very important".

The first part consisted of a set of questions about the demographic information of the participants: gender, age, and years of experience, profession and work department. The second part contained several questions aimed at obtaining information about asthma and its treatment: 1) Do you deal with asthmatic patients?; 2) Do you think mobile health is a complement to the traditional treatment?; 3) Do you support the idea of using smartphone application for patients with asthma during the period of their treatment?; 4) How do you evaluate patients' use of these features: patients profile (patients diagnosis, primary physicians information, satisfaction with the treatment process); push notifications (reminders about the time to take drugs, existence of a weekly or monthly memorandum of treatment with inhalers and other drugs, reminders of clinic appointments and therapy sessions, ask for help notification sending SMS to primary physician about patient's current crisis); disease awareness (pathophysiology of disease, drug guidelines and instructions, drugs side effects, asthma triggers, references such as GINA and SINA); records (data synchronizing with healthcare providers and hospital, medication statistics, number of attacks, visual inputs such as table for peak flow/patient's reading); 5) Why don't you support the idea of using smartphone application for patients with asthma during the period of their treatment? 


\section{Statistical analysis}

The data were expressed in terms of percentages using basic descriptive statistics.

\section{Results}

The demographic information of the respondents of this study is shown in Table 1. In this table we can observe that more than half of the participants (56\%) were males and the majority of them $(96 \%)$ were under 40 years old. In addition, the majority of the respondents $(90 \%)$ were respiratory therapists, and $87 \%$ of the participants were working in respiratory hospital departments.

Also, in Figure 1, we can appreciate that most of the respondents $(96 \%)$ thought that mobile health is a complement to the traditional treatment processes for the purpose of providing medical care remotely. Similarly, according to Figure 2, the majority of the participants (87\%) agreed that mobile treatment is an important part of asthma patients' care.

In Table 2 is presented the evaluation of some features of the patients' profile to be included in the design of a smartphone application for asthma. The data indicated that most of the respondents (98\%) supported the idea that information about patient diagnosis was important or very important to be incorporated in the design of the app. Similarly, the majority of the participants (91\%) believed that patient satisfaction with the therapeutic process, and primary physician accesses $(83 \%)$ were also important or very important. Alike, in Table 3 are described the responses on push notifications in the mobile app. The data showed that $95 \%$ of participants suggested that push notifications about reminder for drugs were important or very important. Likewise, $92 \%$ of the respondents were in favor of having push notification for the existence of a weekly or monthly memorandum of the treatment with inhaler and other drugs. In the same way, $81 \%$ of the respondents considered that having push notifications about reminders of clinic visits and therapy sections were important or very important, and $89 \%$ of them wanted to ask for help sending SMS to primary physician about patients' current crisis.

In Table 4, we observed that the majority of respondents believed that information related to the disease awareness such as asthma triggers, drug guidelines and drug side effects was important or very o important to include in the design of the mobile application. In addition, in Table 5 are illustrated the responses about what data should be needed/recorded for asthma care through the mobile app. The participants believed that the number of asthma attacks is the most important one (98\%) followed by visual inputs about asthma (91\%). As shown in Figure 3, overall, $92 \%$ of the participants considered that an asthma application designed using the guidelines of this study would really help for better asthma care.

\section{Discussion}

In this study, we have proposed the design of an application for the treatment of asthma considering the opinions of a group of healthcare providers from the Kingdom of Saudi Arabia with experience in the management of this disease. It is pertinent to comment that the suggested design is based on accepted clinical and medical practice and on the information and treatment guidelines for this disease; in addition it is the first formal application prepared by our faculty members based on similar research. In this regard, some studies have considered that an asthma application design should be constructed on the opinions of health providers and researchers from universities and hospitals to avoid the limita-

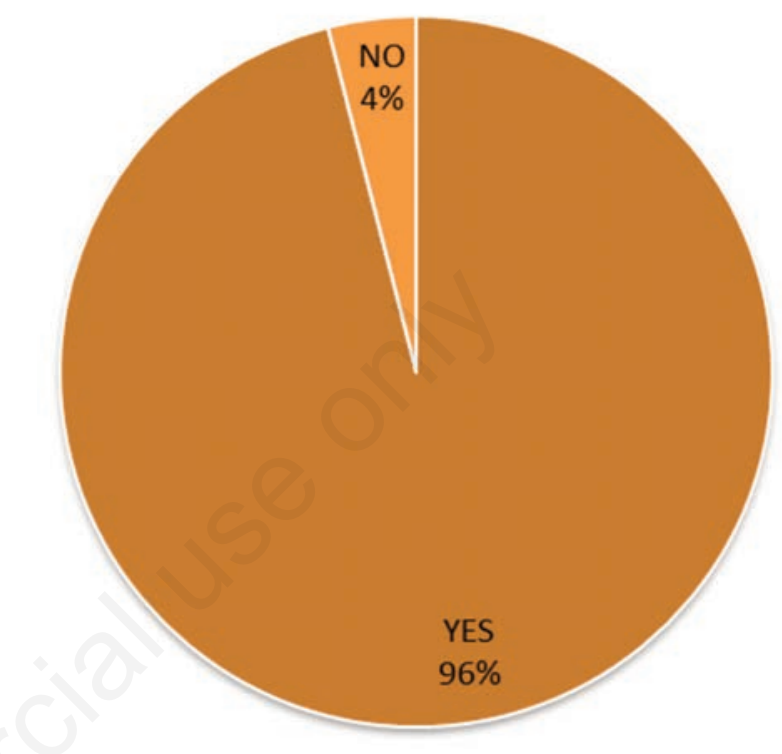

Figure 1. Opinion of respondents about the use of mobile health as a complement to traditional treatment $(n=94)$.

Table 1. Demographic information (n=94).

\begin{tabular}{lcc} 
Gender & n & \% \\
$\quad$ Male & & \\
Female & 53 & 56 \\
Age & 41 & 44 \\
$20-39$ & 32 & \\
$30-39$ & 54 & 55 \\
$40-49$ & 8 & 6 \\
\hline Profession & & \\
Asthma education & 2 & 1 \\
Nurse & 3 & 4 \\
Physician & 5 & 5 \\
Therapist & 84 & 90 \\
Hospital department & & \\
Internal medicine & 1 & 1 \\
Medicine & 2 & 2 \\
Respiratory & 79 & 87 \\
Other & 12 & 10 \\
\hline
\end{tabular}

Table 2. Evaluation of patients' profile features to be included in the app design ( $n=94)$.

\begin{tabular}{lcccc} 
& Not important (\%) & Somewhat important (\%) & Important (\%) & Very important (\%) \\
Patients diagnosis & 0 & 2 & 73 & 25 \\
Primary physician information & 6 & 11 & 47 \\
\hline Satisfaction with the therapeutic process & 1 & 8 & 46 \\
\hline
\end{tabular}


tions of several applications available in the market which are of low quality and have been designed without support or accepted clinical and medical practice and procedures $[4,5,19]$.

According to the opinions of the majority of health providers surveyed in this study, in the design of a useful application for the treatment of patients with asthma it is important or very important to incorporate in the application the following features:

1) Information to increase awareness about the disease, such as asthma triggers, instructions on medications, side effects of

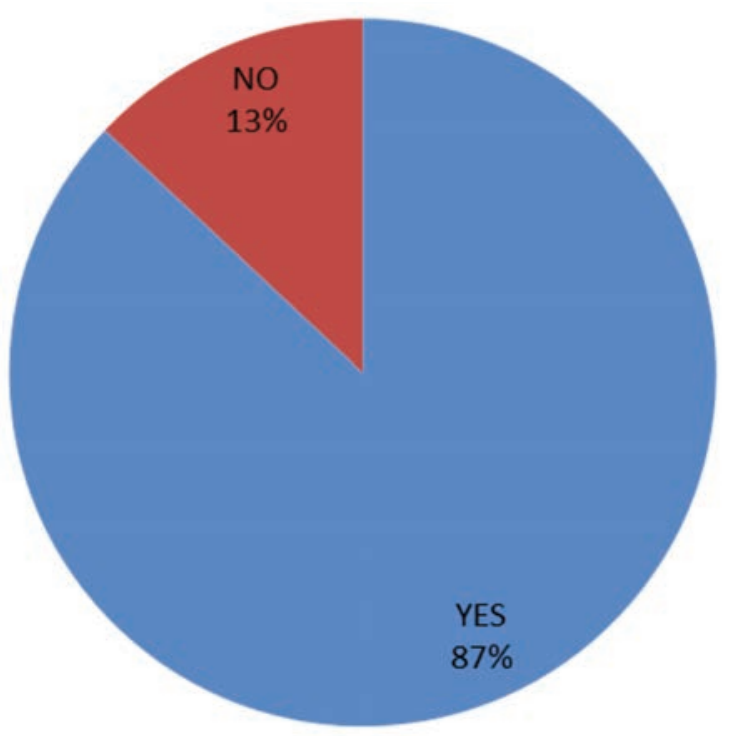

Figure 2. Opinion of respondents about using mobile treatment in asthma patients' care. medications, among other aspects.

2) Information about the patient's profile (patient diagnosis, primary physician attention, and satisfaction with the therapeutic process).

\section{USING OF THIS APPLICATION WILL HELP}

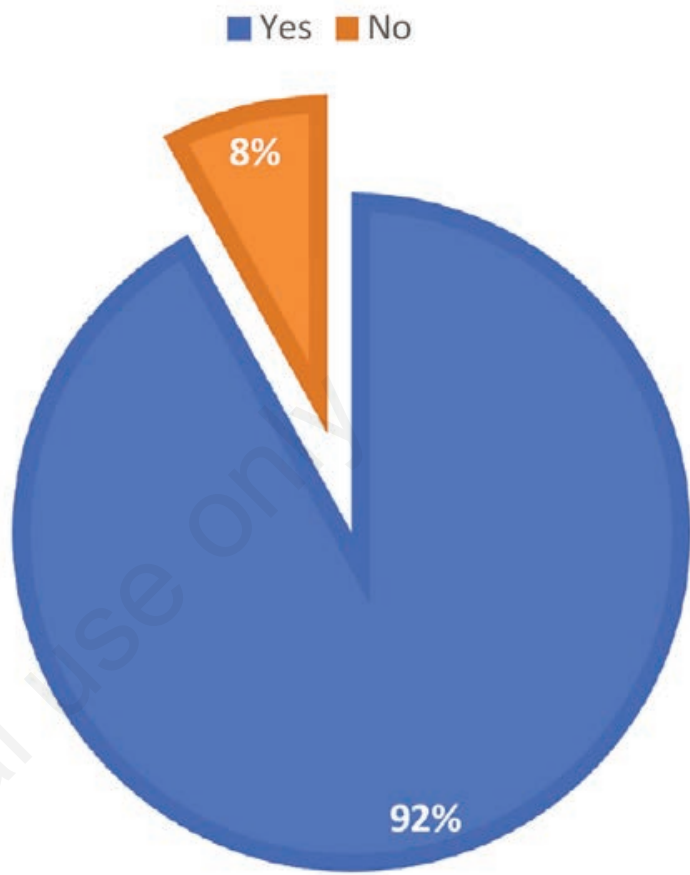

Figure 3. Opinion of respondents about the usefulness of the designed smartphone application for asthma treatment $(n=94)$.

Table 3. Evaluation of push notifications ( $n=94)$.

\begin{tabular}{lccccc} 
& Not important (\%) & Somewhat important (\%) & Important (\%) & Very important (\%) \\
Reminders for drugs & 4 & 1 & 23 & 72 \\
Memorandum of treatment of inhaler and drugs & 0 & 8 & 37 & 31 \\
Reminder of clinic and therapeutic sessions & 1 & 18 & 41 & 50 \\
Ask for help notification & 0 & 11 & 48 \\
\hline
\end{tabular}

Table 4. Evaluation of features related to asthma awareness ( $n=94)$

\begin{tabular}{lcccc} 
& Not important (\%) & Somewhat important (\%) & Important (\%) & Very important (\%) \\
Pathophysiology of disease & 5 & 13 & 51 & 31 \\
Drugs guidelines & 0 & 6 & 36 & 58 \\
Drugs side effects & 0 & 7 & 29 & 27 \\
Asthma triggers & 1 & 1 & 45 & 71 \\
References such as GINA & 12 & 16 & 27 \\
\hline
\end{tabular}

Table 5. Evaluation of information that should be recorded on the asthma app ( $n=94)$.

Not important (\%) $\quad$ Somewhat important (\%) Important (\%) Very important (\%)

Synchronizing with healthcare providers

216

1645

1

$\begin{array}{ll}33 & 53 \\ 40 & 58 \\ 40 & 51\end{array}$


3) Push notifications that alert the patients (reminder for drugs, inhaler, clinic visits, therapy sections), and the responsible doctor or respiratory therapist.

4) To connect (link) the patient directly with his/her healthcare provider(s).

5) To connect (link) the patient with the database in the hospital where he/she receives treatment.

6) To provide the patient with direct steps for treatment in case of an asthma attack until he/she arrives at the hospital or the nearest healthcare center.

7) To provide the patient and the doctor with a timeline (graphics, peak flow) of the number of attacks, the type of medication taken, the time of occurrence of the asthma attack, the activity of the patient before an attack, etc.

It is pertinent to comment that several asthma applications available in the world used some of the characteristics detected in our research, for example, the symptoms of asthma, the recognition of triggers, the medication reminder, the respiration measurements, the peak flow and education about asthma [5,19-20]. However, other features proposed in our design, such as connecting patients directly with their healthcare provider, linking patients to the database of the hospital where they receive treatment, or providing patients with direct steps for treatment in case of an asthma attack until they reach the nearest hospital or health care center, were not observed in existing applications in the market. According to most respondents, the use of an application for asthma with the above distinguishing features would be useful for the treatment of patients with asthma in Saudi Arabia.

The main limitations of this study were the small size of the healthcare providers' sample, and the statistical analysis based only in percentages. Another limitation of this research was that the inferences of the survey are not general and are restricted to the group of the selected participants that responded to the questionnaire. Future work will be directed to overcome these limitations and to target the patients themselves, and then, to link them to the Ministry of Health database in such a way that this model could be a reference throughout the Kingdom of Saudi Arabia, and worldwide in advanced stages. Also, we will investigate, whether the data and conclusions obtained in this research can be extended to the general physician population and if there are differences between the answers of the respiratory therapists' specialists against those of general physicians. In addition, the validation of the App based on asthma clinical trial will be carried out.

\section{Conclusion}

In this research, we have suggested the design of a smartphone application for asthma that, according to the opinion of the majority of healthcare providers surveyed $(92 \%)$, will contribute to improve the treatment of patients with asthma in the Kingdom of Saudi Arabia.

The proposed application based on medical and clinical guidelines allows automatic notifications about medication reminders, clinical visits and therapies; automatic notifications about the weekly or monthly memorandum of treatment with inhaler and other drugs; exhibits information related to the awareness of the disease such as asthma triggers, pharmacological guidelines, side effects of medications and pathophysiology of the disease; presents and transmits in real time data and visual inputs on the history of the number of asthma attacks, peak flow, and medicine statistics; sends SMS to primary physician about patients' current crisis; provides the patient with direct steps for treatment in case of an asthma attack until arrives at the hospital or the nearest healthcare center; delivers useful documentation in real time so that the attending physician knows the patient's condition and can adopt personalized measures appropriate to his/her history and treatment; additionally, allows synchronization with healthcare providers and health centers.

It can be inferred than an App designed with above features could help to decrease the number of cases that need hospitalization, and the number of asthma cases in the emergency departments in the hospitals of the Kingdom. This possibility has a positive impact on public health because it contributes to improve the quality of life of patients, and reduce the costs of treating the disease for both patients and healthcare systems.

\section{Abbreviations}

GINA: Global Initiative for Asthma; ECRHS: European Community Respiratory Health Survey.

\section{References}

1. Global Initiative for Asthma (GINA). 2019 GINA Report, Global Strategy for Asthma Management and Prevention. Accessed on: 17 January 2020. Available at: https://ginasthma.org/gina-reports/

2. Global Asthma Report. The Global Asthma Report 2018. Accessed on: 27 December 2018. Available at: http://www. globalasthmareport.org

3. Istepanian R, Woodward B. m-Health: fundamentals and applications. Wiley-IEEE Press; 2016; 424 pp.

4. Jusoh S. A survey on trend, opportunities and challenges of mHealth apps. iJIM 2017;11.

5. Tinschert P, Jakob R, Barata F, Kramer J, Kowatsh T. The potential of mobile apps for improving asthma self-management: review of publicly available and well-adopted asthma apps. JMIR Mhealth Uhealth 2017;5:e113.

6 . Wu A. The promise of improving asthma control using mobile health. J Allergy Clin Inmunol Pract 2016;4:738-9.

7. Cook KA, Modena BD, Simon RA. Improvement in asthma control using a minimally burdensome and proactive smartphone application. J Allergy Clin Immunol Pract 2016;4:730-7.

8. Alquran A, Lambert K, Farouque A, Holland A, Davies J, Lampugnani E, et al. Smartphone applications for encouraging asthma. Int J Environ Res Public Health 2018;15:2403.

9. Burbank A, Lewis S, Hewes M, Schellhase D, Rettiganti M, Hall-Barrow J, et al. J. Mobile-based asthma action plans for adolescents. J Asthma 2015;52:583-6.

10. Carpenter DM, Geryk LL, Sage A, Arrindell C, Sleath BL. Exploring the theoretical pathways through which asthma app features can promote adolescent self-management. Transl Behav Med 2016;6:509-18.

11. Roberts CA, Geryk LL, Sage AJ, Sleath BL, Tate DF, Carpenter DM. Adolescent, caregiver, and friend preferences for integrating social support and communication features into an asthma self-management app. J Asthma 2016;53:948-54.

12. Al Ghobain MO, Algazlan SS, Oreibi TM. Asthma prevalence among adults in Saudi Arabia. Saudi Med J 2018;39:179-84.

13. World Asthma Day. [Internet]. Accessed on: 27 December 2018. Available at: https://www.moh.gov.sa/Health Awareness/ HealthDay/2018/Pages/HealthDay-2018-05-02.aspx

14. BBC News. Saudi Arabia country profile. [Internet]. Available at: https://www.bbc.com/news/world-middle-east-14702705. Accessed on: 31 December 2018.

15. Statista. [Internet]. Number of smartphone users in Saudi Arabia from 2015 to 2022 (in millions). Accessed on: 31 
December 2018. Available at: https://www.statista.com/statistics/494616/smartphone-users-in-saudi-arabia/

16. General Authority for statistics, Kingdom of Saudi Arabia. Population In Saudi Arabia by Gender, Age, Nationality (Saudi / Non-Saudi) - Mid 2016 A.D. Accessed on: 31 December 2018. Available at: https://www.stats.gov.sa/en/5305

17. Hofer F, Haluza D. Are Austrian practitioners ready to use medical apps? Results of a validation study. BMC Med Inform Decis Mak 2019;19:88.

18. Kamel M, Brewer A, Karimkhani C, Buller D, Dellavalle R.
Mobile medical and health apps: state of the art, concerns, regulatory control and certification. J Public Health Inform 2014; 5:e229.

19. Huckvale K, Morrison C, Ouyang J, Ghaghda A, Car J. The evolution of mobile apps for asthma: an updated systematic assessment of content and tools. BMC Med 2015;13:58.

20. Roberts C, Sage A, Geryk L, Sleath B, Carpenter D. Adolescent preferences and design recommendations for an asthma self-management app: mixed-methods study. JMIR Form Res 2018;2:e10055. 